American Journal of Applied Sciences 3 (12): 2170-2173, 2006

ISSN 1546-9239

(c) 2006 Science Publications

\title{
Development of a Wear Map for the Sliding and Rotational Wear behavior of Shafts
}

\author{
Al-mahasne Mayas Mohammad \\ Mechanical Engineering Department, Tafila Technical University \\ P.O. Box 52 Tafila-Aleas 66141, Jordan
}

\begin{abstract}
This study was an attempt to study the wear calculation method for rotating shaft in the friction couple type shaft-cuff in conditions of oiling in high dusty environment. The size and quantity of abrasive particles that go into the clearance between friction surfaces, definition of the pressure including the load in conjugate that is given by the cuff spring as the radial force, and the effected contact area including the micro hardness of the shaft surface (taking into account the influence of the finishing treatment as a diamond pressing) have been calculated by utilization of above mentioned method. Furthermore, the volume wear has been calculated by the energy of friction including both the factor of energy transition to heat and the latent melting heat of shaft material. In this study the experimental scheme for wear testing of steel shaft in the friction couple type "shaft-cuff" and the theoretical and experimental results have been given, Fig. 4. It has proved that this method permits to choose the effective materials for parts making and the use of effective finishing treatment. Morethan, it permits to choose optimum properties of the parts surface layer. This may be attained on the stage of designing- to prognosis shaft and couple friction conjugates wear. Therefore, this method provides a maximum wear resistance and the ability to calculate parts life before damage.
\end{abstract}

Key words: Calculation method, cuff, friction couple, abrasive particles, abrasive wear

\section{INTRODUCTION}

In conditions of high dusty environment the majority of the friction assemblies in machines working with oil containing the abrasive particles go into the conjugate of friction couples. Therefore the wear resistance of the assemblies decreases intensively.

The method of generating wear resistant surfaces based on advantages of some events accompanying high temperature wear such as oxidation, debris generation and elemental transfer between the contacting surfaces ${ }^{[1-7]}$. Inman et al. ${ }^{[8]}$ investigate the wear behavior during limited debris retention sliding wear of Nimonic 80A versus Stellite 6 (counter face) between room temperature and $750^{\circ} \mathrm{C}$. An earlier study has demonstrated the viability of recycling AZ91 Mg chips via mechanical milling ${ }^{[9]}$. Shanthi et al ${ }^{[10]}$ explore the wear characteristics of recycled AZ91 Mg alloy with grain size in the micron and sub-micron range . Kelly and Arnell ${ }^{[11]}$ investigate the pulsed magnetron sputtering which is a thin film deposition technique in which the magnetron discharge is pulsed from normal operating voltage and ground (unipolar pulsed sputtering) or from the negative operating voltage to positive during the pulse-off periods (bipolar pulsed sputtering). Some works ${ }^{[12-13]}$ present the effect of film residual stresses on the wear behavior of coated systems. Roth et al. ${ }^{[14]}$, and Evans ${ }^{[15]}$ indicate that the wear rates tend to decrease with an increase in the level of compressive stresses, as long as film adhesion is not impaired. Zulai et al. ${ }^{[16]}$ studied the dry-body abrasive wear behavior of the composites to fabricate gray cast iron matrix surface composities reinforced with tungsten carbide particles using vacuum evaporative pattern casting. Shizhong et al. ${ }^{[17]}$ studied the effect of vanadium and carbon on microstructure and abrasive wear resistance of high-speed steel.

Different aspects of abrasive wear and its calculation method of bearing sliding in research projects. However, few investigations have been carried out for conjugates of friction couples type "shaft-cuff". To calculate the abrasive wear it is necessary to know the sizes, quantity of particles being at the same time in the clearance between friction surfaces, definition of the pressure and effected area.

Calculation method: The maximum diameter $\left(\mathrm{d}_{\max }\right)$ of the abrasive particles that go into the clearance was assumed to be equaled the biggest radial friction clearance

$S_{\text {max }}=d_{\text {max }}=2 \cdot\left(\mathrm{R}_{2}-R_{1}\right) \cdot 10^{-3}$

Where, $R_{1}$ - is the radius of shaft neck, mm.

$\mathrm{R}_{2-}$ is the radius of cuff, $\mathrm{mm}$.

The minimum diameter $\left(\mathrm{d}_{\text {min }}\right)$ equals the smallest clearance:

$S_{\text {min }}=d_{\text {min }}=\left(\delta+R_{a 1}+R_{a 2}\right) \cdot 10^{-6}$

where $\delta$ - is the thickness of oiling layer, $\mu \mathrm{m}$.

$R_{a 1}$ and $R_{a 2-}$ are the average values of micro roughness of shaft and cuff respectively, $\mu \mathrm{m}$. 
Therefore, the mean diameter of the particles that follows the frequency distribution of the normal curves equals

$d_{m}=\sqrt{d_{\max } \cdot d_{\text {min }}}$

Then the radius of the abrasive particles is:

$R=\frac{d_{m}}{2}$

The quantity of the abrasive particles passed through the clearance per revolution of the assembly can be defined as follows:

$n_{a}=\frac{\varepsilon \cdot p_{o} \cdot Q \cdot L \cdot K_{1} \cdot K_{2}}{\pi \cdot \omega \cdot p_{a} \cdot R^{3} \cdot R_{1}}$

where $\varepsilon$ is the concentration of the active abrasive particles in oil, \%,

$\mathrm{P}_{\mathrm{o}}$ is the density of the going oil, $\mathrm{kg} / \mathrm{m}^{3}$,

$\mathrm{Q}$-is the volume of oil, going into conjugate per second, $\mathrm{m}^{3} / \mathrm{sec}$,

$\mathrm{L}$ - is the length of conjugate, $\mathrm{m}$,

$\mathrm{K}_{1}=0.85$, is the factor that considers the quantity of particles in oil commensurable with the clearance size ${ }^{[18]}$.

$\mathrm{K}_{2}=0.75-0.95$, is the factor that considers the quantity of particles in oil, hardness of which exceeds the hardness of the friction surfaces ${ }^{[18]}$.

$\Omega$-is the frequency of shaft revolutions, $\mathrm{rad} / \mathrm{sec}$.

$\mathrm{Pa}$ - is the abrasive density, $\mathrm{kg} / \mathrm{m}^{3}$,

$\mathrm{R}$ - is the radius of abrasive particle, $\mathrm{m}$.

The load on each particle is defined by the equation:

$N_{a}=\frac{N}{n_{a}}$

Where, $\mathrm{N}$ - is the load applied to friction assembly, $\mathrm{N}$,

Conjugate load $(\mathrm{N})$ is given by the cuff spring as the radial force generated by spring extension. According to Fig. 1, the load can be defined by the equation:

$N=a R+b$

Where, $\mathrm{R}$ - is the spring radius in the defined moment, $\mathrm{mm}$.

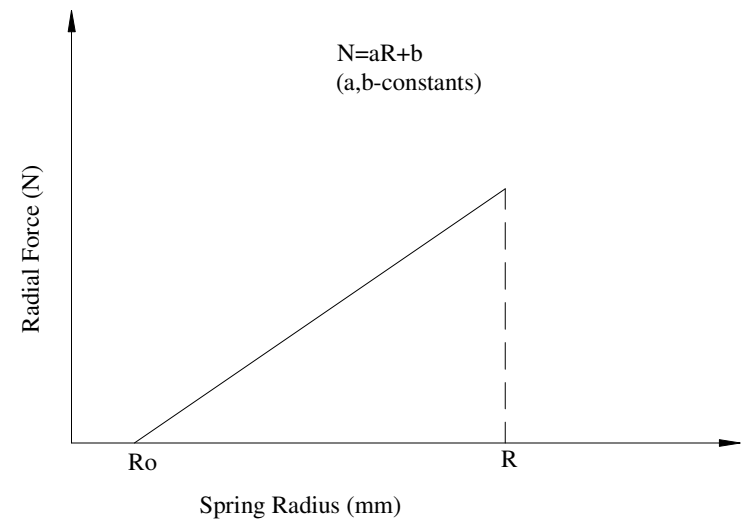

Fig. 1: The relationship between the radial force and the spring radius
Depending on the normal load applied to one of the abrasive particles, depth of the abrasive particle, indentation on the shaft surface is defined by the equation:

$$
h=\frac{N_{a}}{\pi \cdot R \cdot H_{s}}
$$

Where, $\mathrm{H}_{s}, \mathrm{~K}_{\mathrm{s}}$ and $\mathrm{H}-$ is the microhardness of the shaft surface after finish treatment, $\mathrm{Pa}$.

$\mathrm{H}$ - is the microhardness of the shaft.

$\mathrm{K}_{\mathrm{s}-}$ is factor which takes into account the micro hardness after finishing treatment, for example; diamond pressing $\mathrm{K}_{\mathrm{s}}=1.3-2{ }^{[19]}$.

The volume of the abrasive particle indentation on the shaft surface taking it as the spherical segment can be defined by the equation:

$V_{d}=\pi \cdot h^{2} \cdot\left(R-\frac{h}{3}\right)$

Radius of contact area (Fig. 2) can be defined by the equation:

$a=\sqrt{2 R \cdot h-h^{2}}$

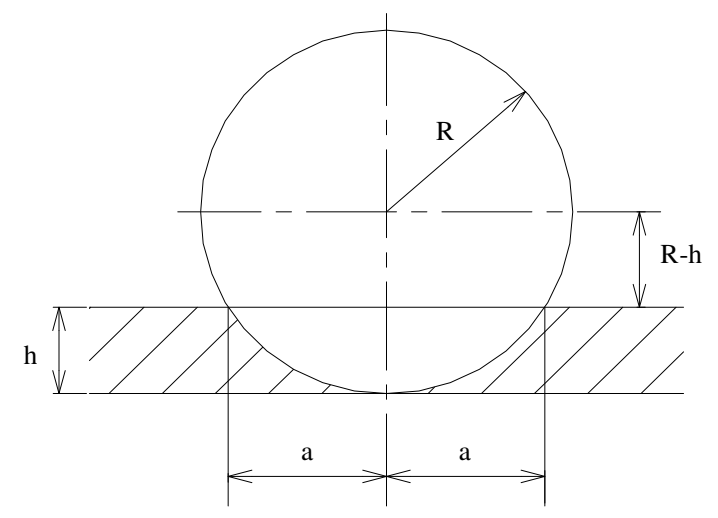

Fig. 2: The scheme of the abrasive particles indentation into the shaft

The real pressure on the contacted surface is defined by the equation:

$P_{r}=\frac{N_{a}}{\pi \cdot a^{2}}$

Elastic and plastic deformations are present at the indentation of the spherical indenture. The contact area in the elastic deformation is defined by the equation:

$$
A=\left(\frac{4 N_{a} \cdot R \cdot(1-\mu)^{2}}{3 \cdot E_{g}}\right)^{0.33}
$$

Where, Eg is the given modulus of elasticity, $\mathrm{M}$ - is the Poisson's factor of the shaft material. The modulus of elasticity is defined by:

$E_{g}=\frac{2 \cdot E_{1} \cdot E_{2}}{E_{1} \cdot E_{2}}$ 
Table 1: Selected properties of pin specimens

\begin{tabular}{|c|c|c|c|c|}
\hline Ductility & $\mathrm{E}(\mathrm{GPa})$ & Hardness (HV) & Grain size $(\mu \mathrm{m})$ & Materials \\
\hline 12.6 & 37.4 & 75.4 & $28.9 \pm 22.5$ & As cast ${ }^{[10]}$ \\
\hline 6.1 & 40.7 & 79.1 & $11.4 \pm 9.3$ & Ball-milled 1h (BM1) ${ }^{[10]}$ \\
\hline 2.8 & 43.8 & 90.2 & $0.6 \pm 10.2$ & Ball-milled $10 \mathrm{~h}(\mathrm{BM} 10)^{[10]}$ \\
\hline 27 & 200 & 540 & $35 \pm 20$ & AISI $N^{\circ} 1045 \mathrm{Q}$ and $\mathrm{T}$ steel (diamond pressing) \\
\hline
\end{tabular}

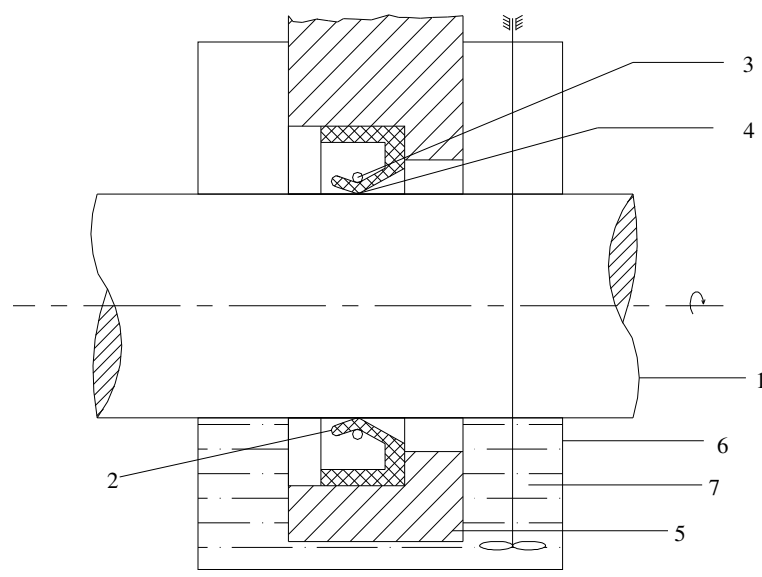

Fig. 3: Experimental scheme for wear testing of steel shaft in the friction couple type, "shaft-cuff", (1- shaft, 2- cuff, 3- spring, 4- conjugate, 5case, 6- Cartier, 7- Lubrication with $0.6 \%$ abrasive particles \{natural sand size 15-55 microns\})

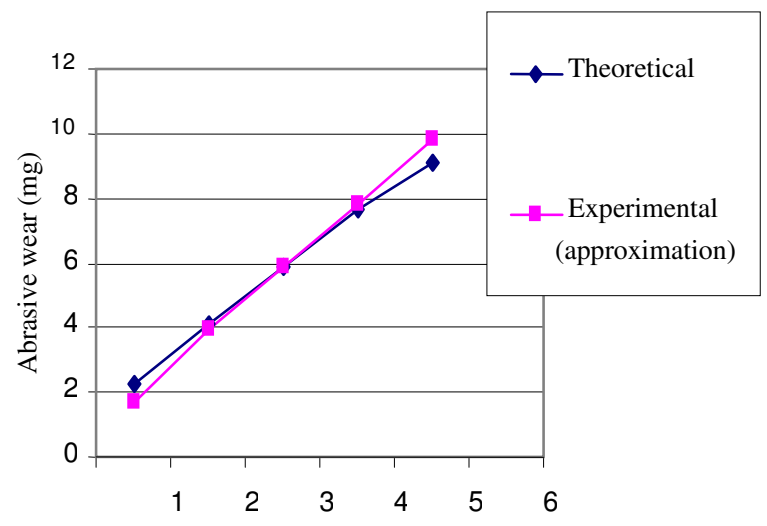

Revolutions of shaft $\times 10^{4}$

Fig. 4: Theoretical and Experimental (after opproximation) abrasive wear results of steel shaft (friction speed $\mathrm{V}==0.339 \mathrm{~m} / \mathrm{sec}$ )

Where $E_{1}$ and $E_{2-}$ are the modulus of elasticity of shaft and cuff materials, respectively.

Energy of elastic deformation friction is defined by the eguation:

$E_{f}=\frac{N_{a} \cdot A^{2}}{2 R}-\frac{2 \cdot A^{3} \cdot V \cdot T \cdot f \cdot P_{r}}{3 R}$

where, $V=\pi \cdot n \cdot R 1 / 30=$ is the speed of sliding (friction speed), $\mathrm{m} / \mathrm{sec}$,

$\mathrm{N}$ - is the shaft rotational speed, r.p.m,

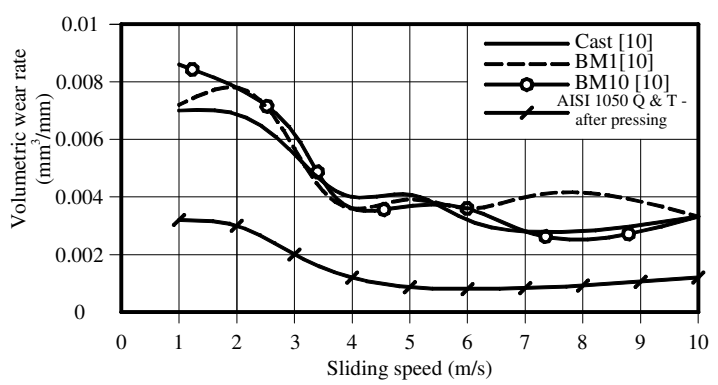

Fig. 5: Variation of wear rate with sliding speed

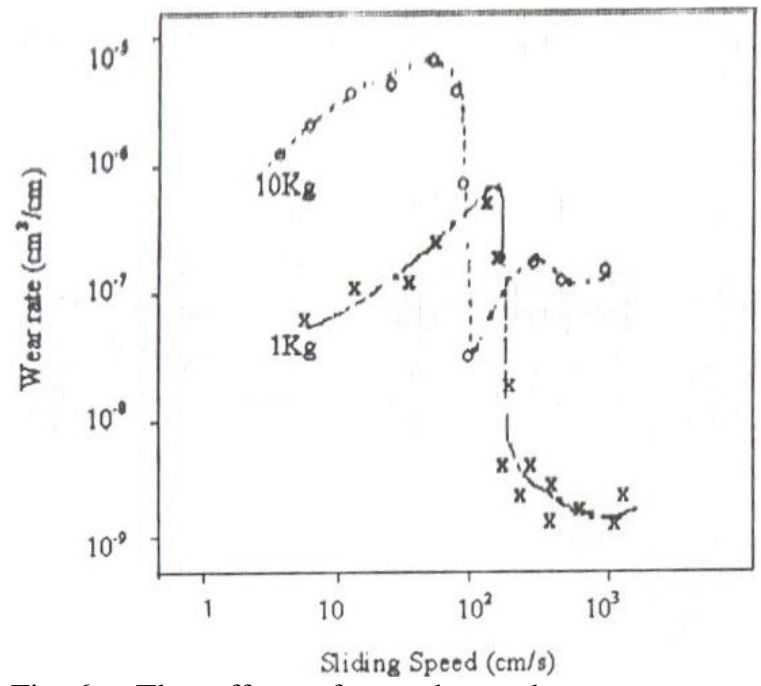

Fig. 6: The effect of speed on the wear rates of medium carbon steel ${ }^{[20,21]}$

$\mathrm{T}$ - is the time, sec,

$f$ - is the coefficient of friction.

Under plastic contact, the energy of friction is defined by the equation:

$E_{f}=\frac{4 \cdot h \cdot V \cdot T \cdot \sigma \cdot\left(2 R \cdot h-h^{2}\right)^{0.5}}{3}+N \cdot h$

Where, $\sigma$ - is the limit of fluidity, Pa.

Initial density of the inner energy is considered a mechanical energy accumulated in metal in the form of the residual tension and for shaft material it equals:

$E_{o}=\frac{(1-2 \mu) \cdot\left(3 \sigma_{r}\right)^{2}}{6 \cdot E_{1} \cdot V_{d}}$

Where, $\sigma_{\mathrm{r}}$ equals the residual tension of shaft surface. The volume wear of shaft is defined by the equation:

$V_{w}=\frac{E_{o} \cdot V_{d} \cdot+(1-K) \cdot E_{f}}{E_{c}}$ 
where $\mathrm{K}=0.88-0.99$ is the factor of energy transition to heat,

Ec- is the latent melting heat of shaft material.

The experimental works done using steel shaft and rubber cuff by the scheme Fig. 3 .

Figure 4 shows both the experimental and theoretical results. It is obvious that approximately experimental and theoretical results are identical. This means that the suggested calculation method can be utilized in the wear calculation for such friction couples and in the calculation of the resource of friction assembles before their damage.

It was found ${ }^{[20,21]}$ that there is a critical sliding speed for medium carbon steel below which continuous severe wear would be encountered and above which a primary stage of severe wear would change to a steady mild wear. Figure 6 shows that wear of medium carbon steel has increased with sliding speed initially. At a load of $1 \mathrm{~kg}$, a maximum wear rate is noted at a speed slightly lower than $1 \mathrm{~m} / \mathrm{s}$. one explanation for sever wear such as this is that at low speeds surface oxide films do not form effectively to provide their protective effect. Although a sort of oxide film could form, the metallic substrate shears readily because of its diminished shear stress at high temperature. This will have the effect of removing both the oxide film and metal. The falling part of the curve with increasing surface speed could be a result of phase change due to flash temperature higher than $800^{\circ} \mathrm{C}$.

\section{CONCLUSION}

It is evident from Fig. $5^{[10]}$ that the wear rates of the three samples (Cast, BM1, BM10) are not distinctly defferent. It is obvious that for quenched and tempered medium carbon steel after diamond pressing, the wear rate is low and continiuos decreasing with increasing the sliding velocity.

The method of abrasive wear calculation of the steel shaft in the friction couple type "shaft-cuff" was given taking into account the changes of micro hardness of the shaft surface after the diamond pressing. This changes the cuff spring radial force, permits already on the stage of designing - to prognosis the shaft and couple friction conjugates wear; choose the optimum properties of the surfaces layer parts to choose the effective materials for parts making, and to use the effective finishing treatment. Therefore, this method provides a maximum wear resistance and the ability to calculate parts life before damage.

\section{REFERENCES}

1. Inman, I.A., 2003. Compacted Oxide Layer Formation under Conditions of Limited Debris Retention at the Wear Interface during high temperature sliding wear of Superalloys, Ph.D. Thesis. Northumbria University, UK.
2. Inman, I.A., S. Datta, H.L. Du, J.S. Burnell-Gray, S. Pierzgalski and Q. Luo, 2003. Microscopy of (glazed) layers formed during high temperature sliding wear at $750^{\circ} \mathrm{C}$. Wear, 254: 461-467.

3. Datta, S., I. Inman, H.L. Du and Q. Luo, 2001. Microscopy of (glazed) layers formed during high temperature wear. Invited Talk at the Institute of Materials, Tribology Meeting, London.

4. Inman, I.A., S. Datta, H.L. Du, J.S. Burnell-Gray, S. Pierzgalski and Q. Luo, 2005. Studies of high temperature sliding wear of metallic dissimilar interfaces, Tribol.

5. Wood, P.D., 1997. The Effect of the counterface on the wear resistance of certain alloys at room temperature and $750^{\circ} \mathrm{C}$. Ph.D. Thesis. Northumbria University, UK.

6. Rose, S., 2000. Studies of the high temperature terminological behavior of some superalloys. Ph.D. Thesis. Northumbria University, UK.

7. Gee, M.G. and N.M. Jennett, 1995. High resolution characterization of tribochemical films on alumina. Wear 193: 133-8.

8. Inman, I.A., S.R. Rose and P.K. Datta, 2006. Development of a simple temperature versus sliding speed wear map for the sliding wear behaviour of dissimilar metallic interfaces, Northumbria University, UK.

9. Lim, S.H., 2002. Mechanical properties of recycled AZ91 chips. B Eng Ehesis. National University of Singapore.

10. Shanthi, M., C.Y.H. lim and L. Lu, 2006. Effect of grain size on the wear of recycled AZ91 Mg. Singapore, Tribology International, 40: 335-338.

11. Kelly, P.J. and R.D. Arnell, 2002. Vacumm, 56: 159.

12. Kattamis, T.Z., M. Chen, S. Skolianos and B.V. Chambers, 1994. Surf. Coat. Technol., 70: 43.

13. Gunnars, J. and A. Alahelisten, 1996. Surf. Coat. Technol., 80: 303.

14. Roth, T.H., K.H. Kloos and E. Broszeit, 1987. Thin. Solid Films 153: 123.

15. Evans, A.G., 1985. Mater. Sci. Eng., 71: 3.

16. Zulai, Li., J. Yehua, Z. Rong, L. Dehong and Z. Rongfeng, 2006. Wear.

17. Shizhong, W., Z. Jinhua and Liujiexu, 2006. Effect of vanadium and carbon on microstructure and abrasive wear resistance of high speed steel. J. Tribol. Intl., 39: 641-648.

18. Lebedov, O.B., 1989. Kemical lubricant materials u special fluids. Tashkent . FAN, pp: 66-67.

19. Torbelo, B.M., 1989. Diamand pressing. Moscow. Machine Bilding, pp: 3, 28-29.

20. Welsh, N.C., 1965. The dry wear of steel, I. Geeneral pattern of behaviour. Phil. Trans. Roy. Soc., London. A257: 31-50.

21. Welsh, N.C., 1965. The dry wear of steel. II. Interpretation and special features, Phil. Trans. Roy. Soc., London, 257: 51-70. 